Olivet Nazarene University

Digital Commons@ Olivet

Faculty Scholarship - Chemistry

Chemistry

$12-2003$

\title{
Quaternary Trialkyl(polyfluoroalkyl)ammonium Salts including Liquid Iodides
}

Om D. Gupta

University of Rajasthan

Douglas Armstrong

Olivet Nazarene University, darmstrg@olivet.edu

Jean'ne M. Shreeve

University of Idaho, jshreeve@uidaho.edu

Follow this and additional works at: https://digitalcommons.olivet.edu/chem_facp

Part of the Materials Chemistry Commons

\section{Recommended Citation}

Gupta, Om D., Paul Douglas Armstrong, and Jean'ne M. Shreeve. "Quaternary Trialkyl(polyfluoroalkyl)ammonium Salts including Liquid Iodides." Tetrahedron Letters 44.52 (2003): 9367-9370.

This Article is brought to you for free and open access by the Chemistry at Digital Commons @ Olivet. It has been accepted for inclusion in Faculty Scholarship - Chemistry by an authorized administrator of Digital Commons @ Olivet. For more information, please contact

digitalcommons@olivet.edu. 
TETRAHEDRON LETTERS

Tetrahedron Letters 44 (2003) 9367-9370 Pergamon

\title{
Quaternary trialkyl(polyfluoroalkyl)ammonium salts including liquid iodides
}

\author{
Om D. Gupta,a Paul Douglas Armstrong ${ }_{\mathrm{b}}$ and Jean'ne M. Shreevec, ${ }^{*}$ \\ aDepartment of Chemistry, University of Rajasthan, Jaipur 302017, India \\ ๖Department of Chemistry, Olivet Nazarene University, Bourbonnais, IL 60914- 2271, USA \\ cDepartment of Chemistry, University of Idaho, Moscow, ID 83844- 2343, USA \\ * Corresponding author.
}

Received 22 July 2003; accepted 30 September 2003

\begin{abstract}
New quaternary ammonium salts that contain polyfluorinated alkyl substituents were prepared by the polyfluoroalkylation of tertiary amines with polyfluorinated alkyl iodides. Metathesis reactions of the iodide salts with fluorine-containing anions resulted in new low melting salts. The iodide salts of the tri(isooctyl)polyfluoroalkyl amines are also liquids at $25^{\circ} \mathrm{C}$.

(c) 2003 Elsevier Ltd. All rights reserved.
\end{abstract}

Despite the large number of tetraalkylammonium salts, only two $\left\{\left(\mathrm{CH}_{3}\right)_{3}\left(\mathrm{CH}_{2} \mathrm{~F}\right) \mathrm{N}_{+} \mathrm{X}-; \mathrm{X}=\mathrm{Br}, \mathrm{I}\right\}$ are known that contain even a single fluorine-containing alkyl substituent.1 Since the report of a liquid ammonium salt, $\mathrm{EtNH}_{3}+\mathrm{NO}_{3}$, , early in the last century, scores of additional quaternary ammonium salts have been synthesized and studied. Typically introduction of greater asymmetry into the packing of the structure by decreasing the symmetry of the cation lowers the melting point.2-9 Very often anions such as trifluoromethanesulfonate or bis(trifluoromethanesulfonyl)amide are employed. In the latter case, there is little opportunity for hydrogen bonding given the greater delocalization of the negative charge over the trifluoromethanesulfonyl groups which contributes to lowering the melting points of the salts markedly. Syntheses of tetraalkylammonium salts via introduction of a smaller anion, such as dicyanamide, results in markedly lower glass transition temperatures as well as reduced viscosities. ${ }_{10}$ While the presence of the thiocyanate anion does not have such a major impact as dicyanamide, liquids with low melting points and high thermal stabilities are also obtained.11 Although some tend to be hygroscopic, tetraalkylammonium salts have the advantage of being less expensive than many of the other core species for preparation of low melting materials with concomitantly high thermal stability.

We now report a series of new water stable low melting salts based on quaternary trialkyl(polyfluoroalkyl)ammonium cations with anions that contain fluorine. Quaternary iodide salts of the commercially available tri(n-propyl), tri(n-butyl) and tri(isooctyl) amines were prepared by reaction with several polyfluorinated alkyl iodides (Scheme 1, Table 1).

The quaternary iodides (1-6) were converted to the corresponding perfluorobutyrates, triflates, or bis(trifluoromethanesulfonyl)amides by metathesis with a slight excess of silver perfluorobutyrate or trifluoromethane sulfonate, or lithium bis(trifluoromethanesulfonyl) amide, respectively (Scheme 1). Thermal stabilities obtained via differential scanning calorimetery for many of the new salts, and density data for the $25^{\circ} \mathrm{C}$ liquids are compiled in Table 1 . These are compared with somewhat similar non-fluorine containing salts from the literature. 3

The quaternary iodides that contain three isooctyl substituents have surprisingly low melting points, that is $<100^{\circ} \mathrm{C}$, e.g. 4-6 are liquids at room temperature. Not unexpectedly the melting points were found to increase

Scheme 1.

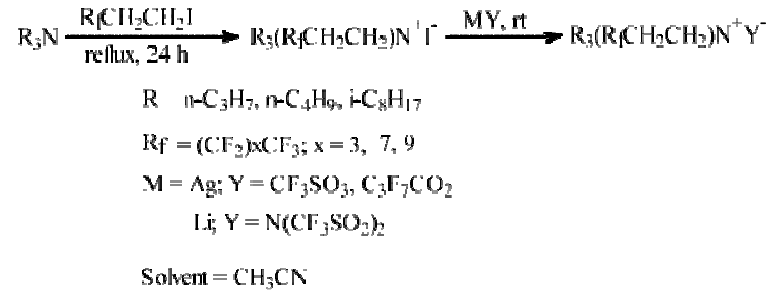


Table 1. Quaternary ammonium salts, $\mathrm{R}_{3}\left(\mathrm{R}_{\mathrm{f}} \mathrm{CH}_{2} \mathrm{CH}_{2}\right) \mathrm{N}_{+} \mathrm{Y}_{-}$

\begin{tabular}{|c|c|c|c|c|c|c|c|}
\hline Compd & $\mathrm{R}$ & $\mathrm{R}_{\mathrm{f}}$ & $\mathrm{Y}$ & Yielda & $\mathrm{db}$ & $T_{m}\left(T_{g}\right)_{c}$ & $\mathrm{~T}_{\mathrm{dd}}$ \\
\hline 1 & $n-\mathrm{C}_{3} \mathrm{H}_{7}$ & $\mathrm{CF}_{3}\left(\mathrm{CF}_{2}\right)_{3}$ & I & 80 & - & 220 & 254 \\
\hline 2 & $n-\mathrm{C}_{4} \mathrm{H}_{9}$ & $\mathrm{CF}_{3}\left(\mathrm{CF}_{2}\right)_{3}$ & I & 80 & - & 82 & 252 \\
\hline 3 & $n-\mathrm{C}_{4} \mathrm{H}_{9}$ & $\mathrm{CF}_{3}\left(\mathrm{CF}_{2}\right)_{7}$ & I & 85 & - & 91 & 260 \\
\hline 4 & $i \mathrm{C}_{8} \mathrm{H}_{17}$ & $\mathrm{CF}_{3}\left(\mathrm{CF}_{2}\right)_{3}$ & I & 83 & 1.1 & 22.5 & 270 \\
\hline 5 & $i-\mathrm{C}_{8} \mathrm{H}_{17}$ & $\mathrm{CF}_{3}\left(\mathrm{CF}_{2}\right)_{7}$ & I & 80 & 1.0 & 20.1 & 265 \\
\hline 6 & $i-\mathrm{C}_{8} \mathrm{H}_{17}$ & $\mathrm{CF}_{3}\left(\mathrm{CF}_{2}\right)_{9}$ & I & 78 & 1.0 & 18.2 & 279 \\
\hline 7 & $n-\mathrm{C}_{3} \mathrm{H}_{7}$ & $\mathrm{CF}_{3}\left(\mathrm{CF}_{2}\right)_{3}$ & OTfe & 85 & - & 110 & 348 \\
\hline \multirow[t]{6}{*}{8} & $n-\mathrm{C}_{4} \mathrm{H}_{9}$ & $\mathrm{CF}_{3}\left(\mathrm{CF}_{2}\right)_{7}$ & $\mathrm{BufO}$ & & 1.4 & $(-48)$ & 345 \\
\hline & $n-\mathrm{C}_{3} \mathrm{H}_{7}$ & $\mathrm{CH}_{3}$ & $\mathrm{NTf}_{2}$ & & - & $105 \mathrm{~h}$ & \\
\hline & $n-\mathrm{C}_{4} \mathrm{H}_{9}$ & $\mathrm{CH}_{3} \mathrm{CH}_{2}$ & $\mathrm{NTf}_{2}$ & & - & $96 \mathrm{~h}$ & \\
\hline & $n-\mathrm{C}_{4} \mathrm{H}_{9}$ & $\mathrm{CH}_{3}\left(\mathrm{CH}_{2}\right)_{5}$ & $\mathrm{NTf}_{2}$ & & 1.12 & $(-63) \mathrm{h}$ & \\
\hline & $n-\mathrm{C}_{4} \mathrm{H}_{9}$ & $\mathrm{CH}_{3}\left(\mathrm{CH}_{2}\right)_{4}$ & $\mathrm{NTf}_{2}$ & & 1.17 & $(-67)_{h}^{h}$ & \\
\hline & $n-\mathrm{C}_{4} \mathrm{H}_{9}$ & $\mathrm{CH}_{3}\left(\mathrm{CH}_{2}\right)_{3}$ & $\mathrm{NTf}_{2}$ & & 1.15 & $26(-68) \mathrm{h}$ & \\
\hline 9 & $n-\mathrm{C}_{4} \mathrm{H}_{9}$ & $\mathrm{CF}_{3}\left(\mathrm{CF}_{2}\right)_{3}$ & $\mathrm{NTf}_{2}$ & 90 & 1.2 & 15.5 & 374 \\
\hline 10 & $n-\mathrm{C}_{4} \mathrm{H}_{9}$ & $\mathrm{CF}_{3}\left(\mathrm{CF}_{2}\right)_{7}$ & $\mathrm{NTf}_{2}$ & 90 & 1.3 & 22.1 & 290 \\
\hline$\underline{11}$ & $i-\mathrm{C}_{8} \mathrm{H}_{17}$ & $\mathrm{CF}_{3}\left(\mathrm{CF}_{2}\right)_{7}$ & $\mathrm{NTf}_{2}$ & 90 & 1.0 & -62.6 & 358 \\
\hline
\end{tabular}

a $\%$.

b $\mathrm{g} / \mathrm{cm}_{3}$, pycnometer; $25^{\circ} \mathrm{C}$.

c Melting point (phase transition) $\left({ }^{\circ} \mathrm{C}\right)$.

d Thermal decomposition $\left({ }^{\circ} \mathrm{C}\right)$

e Triflate.

fHeptafluorobutyrate.

$\mathrm{g}$ Bis(trifluoromethanesulfonyl)amide.

h Ref. 3.

with the decrease in the chain length of either the polyfluoroalkyl or the alkyl group since packing in the crystal lattices would be expected to become more orderly. All of the quaternary iodides are hygroscopic but insensitive to hydrolysis at $25^{\circ} \mathrm{C}$.

In fact, all of these ammonium salts are very stable in air and water at ambient temperature. The solubilities of the salts can be modified by altering the anion or changing the alkyl/polyfluoroalkyl substituent on the ammonium cation. The delocalization of the charge on the amide anion results in a relatively hydrophobic quaternary ammonium salt that separates readily from aqueous solution whereas the reactants and other products are water soluble. This allows straightforward purification of the products via washing with water to remove the impurities. None of the salts is soluble in alkanes. This may be surprising, especially with increasing length of the $\mathrm{R}$ or $\mathrm{R}_{\mathrm{f}}$ group. However, all are soluble to some extent in acetone, dichloromethane, acetonitrile and methanol as determined by observing the qualitative miscibility of each in the referenced solvents.

The usual experimental procedure was as follows. 2- Perfluorobutylethyl iodide (0.75 gm; 2 mmol) was added to a solution of tri( $n$-butyl)amine $(0.37 \mathrm{gm} ; 2 \mathrm{mmol})$ in a $25 \mathrm{~mL}$ flask that contained anhydrous acetonitrile $(10 \mathrm{~mL})$. The mixture was heated at reflux for $24 \mathrm{~h}$ under a dinitrogen atmosphere. Evaporation under vacuum (vacuum line) gave the crude product that was purified by chromatography using a silica gel (70 A_ ) column with an ethyl acetate-acetonitrile (30:70) mixture. The solid material was dried under vacuum. Compound 2 was obtained in $80 \%$ yield. Under similar reaction conditions, the other tertiary amines were converted into analogous iodides (Scheme 1; Table 1, compounds 1-6).12-14

The metathetical reactions with 1-6 were completed with the appropriate anion salt. For example, the liquid iodide (6) (1 mmol) was placed in a $25 \mathrm{~mL}$ round-bottomed flask and dissolved in deionized water $(2 \mathrm{~mL})$. An aqueous solution $(2 \mathrm{~mL})$ of $\mathrm{LiN}\left(\mathrm{SO}_{2} \mathrm{CF}_{3}\right)_{2}(1 \mathrm{mmol})$ was added. The reaction mixture was stirred at $25^{\circ} \mathrm{C}$ for $3 \mathrm{~h}$. The water-soluble product was extracted with ethyl acetate, dried $\left(\mathrm{MgSO}_{4}\right)$, and filtered. Removal of ethyl acetate in vacuo gave the desired product. Compound $\mathbf{1 1}$ was obtained in $90 \%$ yield. Compounds 7-10 were obtained under similar conditions (Scheme 1; Table 1).15-19

Comparing the results as a function of the ammonium cation structure, it has been found that the symmetry of the molecular structure plays an important role in determining their physical properties. However, the thermal stabilities of these salts as determined from differential scanning calorimetric studies seem to vary little with change in substituent on the quaternary ammonium cation, e.g. the iodides are all stable over a range of $252-279^{\circ} \mathrm{C}$. However, with constant anion (iodide) and polyfluoroalkyl substituent, but modifying 
the cation from $n-\mathrm{C}_{3} \mathrm{H}_{7}$ (1) to $n-\mathrm{C}_{4} \mathrm{H}_{9}$ (2) to $i-\mathrm{C}_{8} \mathrm{H}_{17}$ (4) results in a change in the melting point from 220 to 82 to $22.5^{\circ} \mathrm{C}$. The influence of the anion is marked, e.g. a change from iodide in $\mathbf{2}$ to

bis(trifluoromethanesulfonyl) amide in $\mathbf{9}$, with constant $R$, the physical state is changed from solid to liquid at room temperature. The introduction of the polyfluoroalkyl group vis-a'-vis a similar alkyl group (Table 1 ) does not bring about major changes either in density or melting point, e.g. $\left(n-\mathrm{C}_{4} \mathrm{H}_{9}\right)_{3} \mathrm{C}_{6} \mathrm{H}_{13} \mathrm{~N}_{+} \mathrm{NTf}_{2}$ - melts at $26^{\circ} \mathrm{C}\left(d=1.15 \mathrm{~g} / \mathrm{cm}_{3}\right)$ while $\left(n-\mathrm{C}_{4} \mathrm{H}_{9}\right)_{3} \mathrm{C}_{6} \mathrm{~F}_{9} \mathrm{H}_{4} \mathrm{~N}_{+} \mathrm{NTf}_{2}-(9)$ melts only slightly lower at $15.5^{\circ} \mathrm{C}$ $\left(d=1.2 \mathrm{~g} / \mathrm{cm}_{3}\right)$ or $\left(n-\mathrm{C}_{4} \mathrm{H}_{9}\right)_{3} \mathrm{C}_{8} \mathrm{H}_{17} \mathrm{~N}_{+} \mathrm{NTf}_{2}-$ has a $T_{\mathrm{g}}$ at $-63^{\circ} \mathrm{C}\left(d=1.12 \mathrm{~g} / \mathrm{cm}_{3}\right)$ while $\left(n-\mathrm{C}_{4} \mathrm{H}_{9}\right)_{3} \mathrm{C}_{10} \mathrm{~F}_{17} \mathrm{H}_{4} \mathrm{~N}_{+} \mathrm{NTf}_{2}$ -(10) melts at $22.1^{\circ} \mathrm{C}\left(d=1.3 \mathrm{~g} / \mathrm{cm}_{3}\right)$. The lack of effect of fluorinated alkyl groups is somewhat surprising especially given the results we have obtained with the effect of fluorinated substituents in other systems.20 Unfortunately nothing has been reported about the thermal stabilities of the nonfl- uorine-containing tetraalkylammonium salts so no comparison can be made.

In conclusion, new thermally stable liquid salts with trialkylpolyfluoroalkyl quaternary ammonium cations have been synthesized and characterized. Marked variations in melting points appear to be a primary function of the anion and essentially independent of the substituents on the cation. However, when the cation is tri(isooctyl) even the iodide salts are room temperature liquids. Further work is in progress to understand more about physical and chemical properties of this family of new quaternary ammonium salts containing polyfluorinated alkyl groups. Low melting trialkyl ammonium salts with unusual anions will be reported in due course.

\section{Acknowledgements}

The authors are grateful to the National Science Foundation (CHE-0315275) for support of this work. The American Chemical Society Petroleum Research Fund is acknowledged for a summer fellowship for PDA. O.D.G. expresses thanks to the University of Rajasthan, Jaipur, India for leave to carry out this research. Drs. Gary Knerr and Alex Blumenfeld are thanked for mass and NMR spectra, respectively.

\section{References}

1. Bo“hme, H.; Hilp, M. Chem. Ber. 1970, 103, 104-111.

2. Welton, T. Chem. Rev. 1999, 99, 2071-2083.

3. Sun, J.; Forsyth, M.; MacFarlane, D. R. J. Phys. Chem. B 1998, 102, 8858-8864.

4. Sun, J.; Forsyth, M.; MacFarlane, D. R. Molten Salt Forum 1998, 5-6, 585-588.

5. Sun, J.; Forsyth, M.; MacFarlane, D. R. Ionics 1997, 3, 356-362.

6. Ford, W. T.; Hauri, R. J.; Hart, D. J. J. Org. Chem. 1973, 38, 3916-3918.

7. Abbott, A. P.; Capper, G.; Davies, D. L.; Munro, H. L.; Rasheed, R. K.; Tambyrajah, V. Chem. Commun. 2001, 2010-2011.

8. (a) Gordon, J. E.; Subba Rao, G. N. J. Am. Chem. Soc. 1978, 100, 7445-7454; (b) Matsumoto, H.; Yanagida, M.; Tanimoto, K.; Nomura, M.; Kitagawa, Y.; Miyazaki, Y. Chem. Lett. 2000, 922-923.

9. Quinn, B. M.; Ding, A.; Moulton, R.; Bard, A. J. Langmuir 2002, 18, 1734-1742.

10. MacFarlane, D. R.; Golding, J.; Forsyth, S.; Forsyth, M.; Deacon, G. B. Chem. Commun. 2001, 1430-1431.

11. Pringle, J. M.; Golding, J.; Forsyth, C. M.; Deacon, G. B.; Forsyth, M.; MacFarlane, D. R. J. Mater. Chem. 2002 , $12,3475-3480$.

12. Tri-n-butyl(perfluorobutylethyl)ammonium iodide (2): (Yield 80\%), mp $82^{\circ} \mathrm{C}$; IR (KBr): 2936vs, 2874s, 1466vs, 1378s, 1316w, 1235ms, 1104s, 1065ms, 919s, 797s, 736s cm-1; 1 H NMR:_н $\left(200 \mathrm{MHz}, \mathrm{CDCl}_{3}\right): 3.25$ (m, 6H), $3.03(\mathrm{~m}, 2 \mathrm{H}), 2.69(\mathrm{~m}, 2 \mathrm{H}), 1.79(\mathrm{~m}, 6 \mathrm{H}), 1.38(\mathrm{~m}, 6 \mathrm{H}), 0.93(\mathrm{t}, 9 \mathrm{H}) ;{ }_{19} \mathrm{~F} \mathrm{NMR: \_ F}\left(188 \mathrm{MHz}, \mathrm{CDCl}_{3}\right):-80.82(\mathrm{t}, 3 \mathrm{~F})$, $-114.49(\mathrm{~m}, 2 \mathrm{~F}),-124.63(\mathrm{~m}, 2 \mathrm{~F}),-126.27(\mathrm{~m}, 2 \mathrm{~F})$; MS (EI, solid probe), m/e (species, intensity): $432\left(\mathrm{M}_{+}, 25\right)$; HRMS (FAB+) for $\mathrm{C}_{18} \mathrm{H}_{31} \mathrm{NF}_{9}\left(\mathrm{M}_{+}\right)$: calcd 432.2313, found: 432.2320 .

13. Tri-isooctyl(perfluorodecylethyl)ammonium iodide (5): Liquid (Yield, 80\%); IR (KBr, neat): 2958vs, 2929vs, $1463 \mathrm{~s}, 1383 \mathrm{~s}, 1245 \mathrm{~s}, 1019 \mathrm{w}, 895 \mathrm{w}$; cm-1. 1H NMR (300 MHz, $\left.\mathrm{CDCl}_{3}\right) \_$н: 0.92 (t, 9H), 1.24-1.32 (m, 30H), 1.65-1.71 (m, 6H), $2.62(\mathrm{~m}, 2 \mathrm{H}), 3.12(\mathrm{~m}, 2 \mathrm{H}), 3.22(\mathrm{~m}, 6 \mathrm{H}) ;{ }_{19} \mathrm{~F} \mathrm{NMR}\left(188 \mathrm{MHz}, \mathrm{CDCl}_{3}\right) \_$н: $-80.83(\mathrm{t}, 3 \mathrm{~F}),-113.96(\mathrm{~m}, 2 \mathrm{~F})$, $-121.79(\mathrm{~m}, 4 \mathrm{~F}),-122.55(\mathrm{~m}, 4 \mathrm{~F}),-123.24(\mathrm{~m}, 2 \mathrm{~F}),-126.07$ (m, 2F); MS (solid probe) (EI) m/e (\%): $800\left(\mathrm{M}_{+}, 20\right)$; HRMS (FAB+) for $\mathrm{C}_{34} \mathrm{H}_{55} \mathrm{~F}_{17} \mathrm{~N}\left(\mathrm{M}_{+}\right)$: calcd: 800.7903 , found: 800.7935 .

14. Tri-isooctyl(perfluorodecylethyl)ammonium iodide (6): Liquid, (Yield, 78\%); IR (KBr, neat): 2957vs, 2928vs, 2677ms, 1463vs, 1381vs, 1154ms, 1056w; cm-1; 1 H NMR: (200 MHz, CDCl 3$)$ на: 0.87 (t, 9H), 1.26-1.30 (m, 30H), $1.68-1.62(\mathrm{~m}, 6 \mathrm{H}), 2.68(\mathrm{~m}, 2 \mathrm{H}), 3.15(\mathrm{~m}, 2 \mathrm{H}), 3.30(\mathrm{~m}, 6 \mathrm{H}) ;{ }_{19} \mathrm{~F} \mathrm{NMR}\left(188 \mathrm{MHz}, \mathrm{CDCl}_{3}\right) \_\mathrm{F}:-80.09$ (t, $\left.3 \mathrm{~F}\right),-113.96$ (m, 2F), -112.49 (m, 2F), -121.44 (m, 4F), -122 (m, 4F), -123.62 (m, 2F), -124.23 (m, 2F), -127.10 (m, 2F); MS (solid probe) (EI) $\mathrm{m} / z(\%)$ : $900\left(\mathrm{M}_{+}, 15\right)$; HRMS $\left(\mathrm{FAB}_{+}\right)$for $\mathrm{C}_{36} \mathrm{H}_{55} \mathrm{~F}_{21} \mathrm{~N}\left(\mathrm{M}_{+}\right)$: calcd 900.3999 , found: 900.4034 . 15. Tri- $\boldsymbol{n}$-propyl(perfluorobutyl)ethylammonium triflate (7): Yield $85 \%$; mp $110^{\circ} \mathrm{C}$; IR (KBr): $3120 \mathrm{~s}, 2978 \mathrm{vs,}$ 1471s, 1352vs, 1195vs, 1136s, 1059s, 984m, 791m, cm-1; 1 H NMR: (200 MHz, CDCl 3 )_н: 0.93 (t, 9H), 1.87 (m, 6H), $2.64(\mathrm{~m}, 2 \mathrm{H}), 2.72(\mathrm{~m}, 2 \mathrm{H}), 2.97(\mathrm{~m}, 6 \mathrm{H}) ;{ }_{19} \mathrm{~F} \mathrm{NMR}\left(188 \mathrm{MHz}, \mathrm{CDCl}_{3}\right)$ F : -78.62 (s, 3F), -81.16 (t, 3F), -113.72 (m, 2F), -125.18 (m, 2F), -127.12 (m, 2F); MS (solid probe) (EI) $\mathrm{m} / \mathrm{z}(\%): \overline{390}\left(\mathrm{M}_{+}, 12\right)$; Electrospray MS (+ve): $\mathrm{m} / \mathrm{e} 390$ $\left(\mathrm{M}_{+}, 100\right)$; $\left.\mathrm{MS}(-\mathrm{ve}): \mathrm{m} / \mathrm{e} 149\left(\mathrm{CF}_{3} \mathrm{SO}_{3}\right), 100 \%\right)$. 
16. Tri- $\boldsymbol{n}$-butyl(perfluorooctylethyl)ammonium perfluorobutyrate (8): Liquid (Yield, $80 \%$ ); IR (KBr, neat): 2960vs, 2876vs, 2740m, 1681vs, 1467s, 1382s, 1330s, 1222vs, 1114m, 962ms, 926m, 804m, cm-1; 1H NMR: (200 $\left.\mathrm{MHz}_{2} \mathrm{CDCl}_{3}\right) \_$н: $0.94(\mathrm{t}, 9 \mathrm{H}), 1.31(\mathrm{~m}, 6 \mathrm{H}), 1.85(\mathrm{~m}, 6 \mathrm{H}), 2.82(\mathrm{~m}, 2 \mathrm{H}), 3.17(\mathrm{~m}, 2 \mathrm{H}), 3.31(\mathrm{~m}, 6 \mathrm{H})$; ${ }_{19} \mathrm{~F} \mathrm{NMR:} \mathrm{(188}$ $\mathrm{MHz}_{\mathrm{CDCl}} \mathrm{CD}_{-} \mathrm{F}:-80.58(\mathrm{t}, 3 \mathrm{~F}),-81.72(\mathrm{t}, 3 \mathrm{~F}),-112.61(\mathrm{~m}, 4 \mathrm{~F}),-117.37(\mathrm{~m}, 2 \mathrm{~F}),-121.69(\mathrm{~m}, 6 \mathrm{~F}),-123.21(\mathrm{~m}, 4 \mathrm{~F})$, -126. $22(\mathrm{~m}, 2 \mathrm{~F})$; MS (solid probe) (EI) $\mathrm{m} / z(\%)$ : $632\left(\mathrm{M}_{+}, 16\right)$; Electrospray MS (+ve): $\mathrm{m} / \mathrm{e} 632\left(\mathrm{M}_{+}, 70 \%\right)$; MS (-ve): $m / e 212\left(\mathrm{C}_{3} \mathrm{~F}_{7} \mathrm{COO}_{-}, 70 \%\right)$.

17. Tri- $\boldsymbol{n}$-butyl(perfluorobutylethyl)ammonium bistrifluoromethanesulfonylamide (9): Liquid (Yield 90\%); IR (KBr, neat): 3143s, 2966vs, 2878ms, 1467ms, 1351s, 1193vs, 1136m, 924m, 789m, cm-1; 1H NMR (200 MHz, CDCl 3 ) _н: $0.97(\mathrm{t}, 9 \mathrm{H}), 1.29(\mathrm{~m}, 6 \mathrm{H}), 1.75(\mathrm{~m}, 6 \mathrm{H}), 2.71(\mathrm{~m}, 2 \mathrm{H}), 3.12(\mathrm{~m}, 2 \mathrm{H}), 3.22(\mathrm{~m}, 6 \mathrm{H}) ;{ }_{19} \mathrm{~F} \mathrm{NMR}(188$ O. D. Gupta et al. /Tetrahedron Letters 44 (2003) 9367-9370 $\left.9370 \mathrm{MHz}, \mathrm{CDCl}_{3}\right)$ _F: -79.52 (s, 6F), -80.75 (t, 3F), -114.52 (m, 2F), -124.59 (m, 2F), -126.17 (m, 2F); MS (solid probe) (EI) m/z (\%): $432\left(\mathrm{M}_{+}, 17\right)$; Electrospray MS (+ve): $m / e$ 432 (M+, 100\%); MS (-ve): $m / e 280\left(-\mathrm{N}\left(\mathrm{CF}_{3} \mathrm{SO}_{2}\right)_{2}, 100 \%\right)$.

18. Tri- $\boldsymbol{n}$-butyl(perfluorooctylethyl)ammonium bistrifluoromethanesulfonylamide (10): Liquid (Yield 90\%); IR (KBr, neat): 3138ms, 2966vs, 2878ms, 1467s, 1351s, 1194bs, 1136m, 1059ms, 924m, 823m, cm-1; 1 H NMR (200 $\left.\mathrm{MHz}_{1} \mathrm{CDCl}_{3}\right) \_$н: $0.96(\mathrm{t}, 9 \mathrm{H}), 1.31(\mathrm{~m}, 6 \mathrm{H}), 1.78(\mathrm{~m}, 6 \mathrm{H}), 2.78(\mathrm{~m}, 2 \mathrm{H}), 3.17(\mathrm{~m}, 2 \mathrm{H}), 3.27$ (m, 6H); $19 \mathrm{~F}$ NMR (188 $\mathrm{MHz}_{\mathrm{CDCl}} \mathrm{CD}_{\mathrm{F}} \mathrm{F}-79.42(\mathrm{~s}, 6 \mathrm{~F}),-80.65(\mathrm{t}, 3 \mathrm{~F}),-112.58(\mathrm{~m}, 2 \mathrm{~F}),-122.18(\mathrm{~m}, 2 \mathrm{~F}),-122.78(\mathrm{~m}, 4 \mathrm{~F}),-123.62(\mathrm{~m}, 2 \mathrm{~F})$, $-124.23(\mathrm{~m}, 2 \mathrm{~F}),-126.58(\mathrm{~m}, 2 \mathrm{~F})$; MS (solid probe) (EI) $\mathrm{m} / \mathrm{z}(\%): 632\left(\mathrm{M}_{+}, 2\right)$; Electrospray MS (+ve): $\mathrm{m} / \mathrm{e} 632$ $\left(\mathrm{M}_{+}, 100 \%\right)$; $\mathrm{MS}(-\mathrm{ve}):$ m/e $\left.280\left(-\mathrm{N}\left(\mathrm{CF}_{3} \mathrm{SO}_{2}\right)_{2}\right), 100 \%\right)$.

19. Tri-isooctyl(perfluorooctylethyl)ammonium bis(trifluoromethanesulfonyl) amide (11): Liquid (Yield 90\%); IR (KBr, neat): 2959vs, 2729w, 1463s, 1358vs, 1180vs, 1135s, 965m, 788m, 740m, cm-1; $1 \mathrm{H}$ NMR;_н (200 MHz, $\left.\mathrm{CDCl}_{3}\right): 3.28(\mathrm{~m}, 6 \mathrm{H}), 3.18(\mathrm{~m}, 2 \mathrm{H}), 2.62(\mathrm{~m}, 2 \mathrm{H}), 1.62-1.59(\mathrm{~m}, 6 \mathrm{H}), 1.22-1.29(\mathrm{~m}, 30 \mathrm{H}), 0.84(\mathrm{t}, 9 \mathrm{H})$; ${ }_{19} \mathrm{~F}$ NMR: $\mathrm{F}$ $\left(188 \mathrm{MHz}, \mathrm{CDCl}_{3}\right)$ : -75.12 (s, 6F), -82.04 (t, 3F), -113.76 (m, 2F), -122.28 (m, 2F), -122 (m, 4F), -123.52 (m, 2F), -124.13 (m, 2F), $-127.10(\mathrm{~m}, 2 \mathrm{~F})$, MS (EI) m/e (species, intensity): $800\left(\mathrm{M}_{+}, 20\right)$; HRMS (FAB $\left.{ }_{+}\right)$for $\mathrm{C}_{34} \mathrm{H}_{55} \mathrm{~F}_{17} \mathrm{~N}\left(\mathrm{M}_{+}\right)$: calcd 800.7903, found: 800.7935 .

20. (a) Mirzaei, Y. R.; Twamley, B.; Shreeve, J. M. J. Org. Chem. 2002, 67, 9340-9345; (b) Singh, R. P.; Manandhar, S.; Shreeve, J. M. Tetrahedron Lett. 2002, 43, 9497-9499; (c) Mirzaei, Y. R.; Shreeve, J. M. Synthesis 2002, 24-26; (d) Singh, R. P.; Manandhar, S.; Shreeve, J. M. Synthesis 2003, 1579-1585. 